\title{
Características da população ribeirinha de um município do interior do Amazonas
}

Characteristics of the riverside population of a municipality in the interior of the Amazon

Características de la población de río de un municipio en el interior de la Amazonía

Marcelo Henrique da Silva Reis ${ }^{1 *}$, Jéssica Karoline Alves Portugal ${ }^{1}$, Genice Lemos Campos $^{2}$, Vanderson de Souza Pereira ${ }^{1}$, José Carlos Ferreira Pinheiro Júnior ${ }^{1}$, Sibele Naiara Ferreira Germano1, Tanny Thaylle Gomes de Souza1 ${ }^{1}$ Évelyn Janaína da Silva Barão ${ }^{1}$, Nathalie Marinho Freire $^{1}$, Yara da Silva dos Reis ${ }^{1}$.

\section{RESUMO}

Objetivo: Relatar as características da população ribeirinha através da vivência e experiência adquirida durante o período de 4 anos por gestores da saúde ribeirinha de um município do interior do Amazonas. Relato de experiência: Trata-se de um relato de experiência do tipo descritivo, obtido com o conhecimento e vivências adquiridos durante 4 anos de gestão profissional envolvendo o cuidado das populações ribeirinhas de um município do interior do Amazonas, entre 2017 a 2021. Neste período, foi possível acompanhar diariamente todos os planejamentos e desenvolvimento de atividades realizados na zona rural local. Ao longo dos últimos 4 anos, foram realizadas 40 viagens pela UBS Fluvial, neste período foram alcançadas todas as 206 comunidades locais, contemplando todas as regiões, com a realização de aproximadamente 60.000 atendimentos de diversas especialidades. Considerações finais: Considerando as peculiaridades das extensas áreas rurais do município, sugere-se o estudo da viabilidade econômica da UBS fluvial e a possibilidade de implantação de polos bases de saúde, similares ao modelo implantado em Distritos Sanitários Indígenas (DSEI), possibilitando atendimento contínuo e talvez, a redução dos custos na operacionalização das ações de saúde.

Palavras chave: População rural, Atenção primária à saúde, Saúde pública.

\begin{abstract}
Objective: To report the characteristics of the riverside population through the experience and experience acquired during a period of 4 years by riverside health managers in a municipality in the interior of Amazonas. Experience report: This is a descriptive experience report, obtained from the knowledge and experiences acquired during 4 years of professional management involving the care of riverside populations in a municipality in the interior of the Amazon, between 2017 and 2021. It was possible to monitor on a daily basis all the planning and development of activities carried out in the local rural area. Over the last 4 years, 40 trips were made by UBS Fluvial, in this period, all 206 local communities were reached, covering all regions, with approximately 60,000 visits in various specialties. Final considerations: Considering the peculiarities of the city's extensive rural areas, it is suggested to study the economic feasibility of the fluvial UBS and the possibility of implementing health base centers, similar to the model implemented in Indigenous Health Districts (DSEI), enabling continuous care and perhaps, the reduction of costs in the operationalization of health actions.
\end{abstract}

Keywords: Rural population, Primary health care, Public health.

${ }_{1}^{1}$ Universidade Federal do Amazonas (UFAM), Coari - AM. *E-mail: reis.henrique.marcelo@gmail.com

${ }^{2}$ Centro Universitário do Norte (UNINORTE), Manaus - AM.

SUBMETIDO EM: 11/2021

ACEITO EM: 11/2021

PUBLICADO EM: 11/2021 


\section{RESUMEN}

Objetivo: Informar las características de la población ribereña a través de la experiencia y experiencia adquirida durante un período de 4 años por los gestores de salud ribereña en un municipio del interior del Amazonas. Informe de experiencia: Se trata de un informe descriptivo de experiencia, obtenido a partir de los conocimientos y experiencias adquiridos durante 4 años de gestión profesional que involucran el cuidado de poblaciones ribereñas en un municipio del interior de la Amazonía, entre 2017 y 2021., fue posible monitorear en el día a día toda la planificación y desarrollo de las actividades que se realizan en el ámbito rural local. En los últimos 4 años se realizaron 40 viajes por UBS Fluvial, en este período se llegó a las 206 comunidades locales, abarcando todas las regiones, con aproximadamente 60.000 visitas en diversas especialidades. Consideraciones finales: Considerando las peculiaridades de las extensas áreas rurales de la ciudad, se sugiere estudiar la viabilidad económica de la UBS fluvial y la posibilidad de implementar centros de base de salud, similar al modelo implementado en los Distritos de Salud Indígenas (DSEI), que permitan una atención continua. y quizás, la reducción de costos en la operacionalización de acciones de salud.

Palabras clave: Población rural, Atención primaria a la salud, Salud pública.

\section{INTRODUÇÃO}

As populações ribeirinhas que vivem no Estado do Amazonas possuem diversas peculiaridades em relação à população urbana local. Dentre as diferenças, podemos destacar: exposição às doenças tropicais, isolamento geográfico, desde minutos a dias de distância, fatores que podem levar a exclusão social e consideráveis limitações de acesso aos serviços de saúde, neste contexto, esses empecilhos significam um grande desafio para ações de saúde pública, especialmente nas áreas ribeirinhas dos municípios do interior (REIS MHS, et al., 2019).

Os ribeirinhos desta região são caracterizados por viverem em comunidades geralmente distantes das sedes municipais, sendo compostas por núcleos familiares, distribuídas ao longo das margens de rios, lagos e igarapés. Essa população representa uma mistura de diferentes grupos sociais: indígenas, nordestinos, migrantes de outras regiões, dentre outros (DIEGUES AC, et al., 2000; FRAXE TJP, et al., 2007).

Os comunitários residentes nestas áreas utilizam a floresta e os rios para garantir a sobrevivência, seja com a venda ou consumo próprio. A principal fonte de alimentos vem da pesca e agricultura, sendo os moradores da região ribeirinha local privilegiados, pois o município possui um vasto manancial de peixes diversos e frutos variados, que fazem parte do calendário nutricional dos ribeirinhos. Esses alimentos possuem grande valor para a nutrição dos comunitários, além de estarem acessíveis na maior parte do ano. Dentre os produtos disponíveis, podemos destacar: a mandioca, que é utilizada para fabricação de farinha, goma, tucupi e beiju; cheiro verde, cebola de palha, couve, alface, pepino, tomate, macaxeira, banana, melancia, melão, castanha, açaí, pupunha, tucumã, dentre outros alimentos (DIEGUES AC, et al., 2000; FRAXE TJP, et al., 2007; FRANCO EC, et al., 2015).

A economia dos ribeirinhos é movida pela venda do pescado e também dos produtos agrícolas. A sua comercialização é realizada na própria comunidade, sendo vendidas a terceiros, que compram grandes quantidades com valores abaixo do mercado e posteriormente revendem com grande porcentagem em cima do valor inicial, além disto, os ribeirinhos agricultores também realizam vendas nas grandes feiras locais (REIS MHS, et al., 2019).

Ainda em relação a situação econômica, a renda dessas populações é complementada por recursos advindos de programas do governo federal e municipal, com destaque para o Programa Bolsa Família e Direito à Cidadania (GUIMARÃES AF, et al., 2020). Além disto, os ribeirinhos que têm a pesca como fonte de renda, recebem o seguro defeso (benefício dado em períodos do ano em que a pesca comercial é proibida por decreto, devido a reprodução dos peixes). Este seguro é garantido por lei, durante cinco meses, com intuito de assegurar a preservação da biodiversidade local de espécies (CAMPOS AG, CHAVES JV, 2014). 
Apesar da inserção do Programa Federal Luz para todos, a distribuição de energia elétrica ainda é insuficiente, muitas comunidades foram agraciadas com o programa, porém, observa-se que a maioria das residências possui eletricidade instável e necessitam do suporte de geradores de energia, que funcionam a base de combustíveis. O principal meio de transporte utilizado na zona rural são as canoas de madeira ou alumínio, que possuem acoplada a suas estruturas motores conhecidos como "rabetas" e motor de popa, que dependendo da situação financeira do proprietário pode variar de potência, visto que quanto maior a potência mais caro o motor (GAMA ASM, 2016).

Em relação as condições de moradia, em sua maioria, as casas são feitas de madeira (palafitas), localizadas de forma suspensa devido aos períodos de cheia, além de casas flutuantes, construídas com as mesmas características das casas de madeira, contando com o auxílio de grandes troncos de árvores (açacu), de modo que possam funcionar como boias e facilitar a flutuação dessas residências (GAMA ASM, et al., 2018).

Diante do exposto, o objetivo deste estudo foi relatar as características da população ribeirinha através da vivência e experiência adquirida durante o período de 4 anos por gestores da saúde ribeirinha de um município do interior do Amazonas.

\section{RELATO DE EXPERIÊNCIA}

Trata-se de um relato de experiência do tipo descritivo, obtido com o conhecimento e vivências adquiridos durante 4 anos de gestão profissional envolvendo o cuidado das populações ribeirinhas de um município do interior do Amazonas, entre 2017 a 2021. Neste período, foi possível acompanhar diariamente todos os planejamentos e desenvolvimento de atividades realizados na zona rural local.

O município localiza-se na parte central do estado, estando distante a mais de $350 \mathrm{~km}$ da capital. O seu acesso pode ser feito por via área, com viagens entre 60 a 85 minutos e também por via fluvial, que varia entre 9 a 30 horas. Em relação ao quantitativo populacional, de acordo com o último censo realizado, estimase que a população local seja de 75.965 habitantes (INSTITUTO BRASILEIRO DE GEOGRAFIA E ESTATÍSTICA (IBGE), 2010).

Grande parte das comunidades ribeirinhas possuem igrejas e centros comunitários, locais que são utilizados para celebrações, reuniões ou confraternizações em datas festivas, principalmente dos padroeiros das comunidades. Uma liderança local é escolhida democraticamente pela população residente, mediante a votação entre os comunitários, cabendo ao vencedor representar a comunidade em reuniões no município, além de buscar resoluções dos principais problemas encontrados pelos comunitários, e caso não esteja atuando de forma satisfatória, uma nova eleição é realizada para definir um representante mais participativo e resolutivo.

Esse mesmo modelo de eleição também é utilizado para a escolha do Agente Comunitário de Saúde (ACS), porém, para participar da eleição é necessário ter o ensino médio como escolaridade mínima. Os três mais votados pelo público passarão posteriormente por uma prova de conhecimentos gerais da profissão, elaborada pelo Departamento de Atenção Básica do município, para que assim o maior pontuador desta prova possa ser treinado e passe a exercer a função segundo as atribuições impostas pela Política Nacional de Atenção Básica (PNAB).

A estrutura municipal no que tange os serviços de saúde disponíveis estão distribuídos da seguinte maneira: Departamento de Vigilância em Saúde, responsável por todas as ações de vigilância do município; Departamento de Atenção básica, que contempla 13 Unidades Básicas de Saúde espalhadas pelo território local e 1 Unidade Básica de Saúde Fluvial; Serviços de média complexidade, que incluem: Hospital Regional com mais de 100 leitos disponíveis; Instituto de Medicina Tropical; Laboratório de análises clínicas, ofertando milhares de exames mensalmente, Serviço de Emergência com suporte de 3 ambulâncias; Policlínica que oferta atendimentos especializados; Centro Especializado em Reabilitação e um Centro de Atenção Psicossocial (CAPS) (REIS MHS, et al., 2019). 
Com a criação do Sistema Único de Saúde - SUS, houve o processo de descentralização da saúde. Desta maneira, criou-se o Programa de Agentes Comunitários de Saúde (PACS), inspirado na prevenção de doenças por ações desenvolvidas pelos Agentes Comunitários de Saúde (ACS). O ACS atua em dois importantes programas do Ministério da Saúde: o PACS e a Estratégia Saúde da Família (ESF), que caracterizam a municipalização e descentralização das ações de atenção primária à saúde no Brasil (MINISTÉRIO DA SAÚDE, 2001).

O cuidado à saúde ribeirinha é realizado com embasamento na PNAB, que ao longo dos anos foi criando e retificando portarias. Neste sentido, a implementação das portarias MS/GM oㅡ 2.488 e 2.490, ambas de 2011 (MINISTÉRIO DA SAÚDE, 2011a; MINISTÉRIO DA SAÚDE, 2011b), possui grande relevância para o contexto ribeirinho. Através desses documentos é direcionado os arranjos disponíveis para ofertar atendimento a quem reside em zonas rurais, além dos arranjos existentes para o resto do território nacional (MINISTÉRIO DA SAÚDE, 2012).

As ações desenvolvidas atualmente pelos profissionais nas comunidades ribeirinhas são: Consulta Médica; Consulta de Enfermagem contemplando todas as ofertas impostas pelo Ministério da Saúde; Consultas e procedimentos odontológicos; Procedimentos de enfermagem conforme necessidade; Educação em Saúde; Busca ativa de doenças e agravos não transmissíveis; Visitas domiciliares e orientações individualizadas; Planejamento familiar; Programa Saúde na Escola; Realização de exames laboratoriais; Reuniões com a comunidade visando o planejamento, monitoramento e avaliação das ações desenvolvidas e Ações de vigilância (REIS MHS, et al., 2020).

Ao longo dos últimos 4 anos, foram realizadas 40 viagens pela UBS Fluvial, neste período foram alcançadas todas as 206 comunidades locais, contemplando todas as regiões, com a realização de aproximadamente 60.000 atendimentos de diversas especialidades.

\section{DISCUSSÃO}

O Brasil é um país que possui sistema de saúde gratuito, sem distinção de raça, cor, classe social, sendo capaz de chegar as regiões mais longínquas de nosso território. O Sistema Único de Saúde (SUS) é o único capaz de alcançar as populações mais isoladas geograficamente, sejam ribeirinhos, indígenas ou quilombolas, proporcionando a elas um de seus princípios mais importantes que é a equidade e acesso universal às políticas de saúde (BRASIL, 1990).

Mediante a dificuldade de acesso aos serviços de saúde, os comunitários contam com o conhecimento empírico quando precisam cuidar da saúde, além do apoio do ACS da comunidade, visto que os serviços de saúde estão a horas de distância. Neste sentido, estes cidadãos recebem assistência de saúde nas seguintes situações: 1) quando procuram a sede do município; 2) na passagem de barcos missionários de Organizações Não Governamentais (ONG) ou quando a equipe de saúde através do Barco da Saúde ou Unidade Básica de Saúde Fluvial (UBSF) desloca-se até sua comunidade.

Os ACS são personagens importantíssimos no contexto da saúde ribeirinha, possuindo características peculiares, considerando que além de trabalhar, devem residir na comunidade, tornando mais forte a relação entre trabalho e vida social, estabelecendo vínculo contínuo com seus assistidos. Ao longo dos anos, ocorreu a inserção de novas atribuições a estes profissionais, conforme a nova atualização da PNAB, além das atribuições que já faziam parte do ciclo de trabalho, com ênfase na prevenção de doenças e promoção da saúde, por meio de ações educativas individuais e coletivas, realizadas nos domicílios e comunidade. Dentre elas, destaca-se o cadastro/diagnósticos; mapeamento; identificação das microáreas de risco; realização de visitas domiciliares; ações coletivas e intersetoriais (MINISTÉRIO DA SAÚDE, 2017).

Na zona rural não há rede de esgoto e estação de tratamento da água consumida, em sua grande maioria, os ribeirinhos utilizam fossas rudimentares para suas necessidades fisiológicas e posteriormente o despejo dos dejetos (SOUSA IS, 2009). No caso das casas flutuantes e outras residências que não contam com fossas, os dejetos são lançados diretamente nos rios, neste caso, é importante salientar que o rio ou lago onde os dejetos são despejados é o mesmo que a maioria dos indivíduos utiliza para captar água para consumo, lavagem e preparo dos seus alimentos (GAMA ASM, 2016). 
A falta de informação e baixo nível educacional pode estar atrelado a essas práticas. De acordo com informações repassadas pelos ACS durante as reuniões mensais, os índices de doenças enteropasitárias representa uma das principais causas dos problemas de saúde envolvendo ribeirinhos, principalmente as crianças, corroborando com outro estudo realizado no mesmo município (SANTOS FS, et al., 2010). Diante da problemática, a Secretaria Municipal de Saúde tem focado em capacitações e fornecimento satisfatório de hipoclorito de sódio para tratamento adequado da água consumida, a fim de reduzir os índices de verminoses entre essa população.

Estar próximo da floresta amazônica e do clima tropical, constituem-se como fatores relevantes para a ocorrência de agravos típicos da região ao caboclo ribeirinho. Nas áreas rurais é rotineiro o surgimento de casos de malária, acidentes por animais peçonhentos como cobras, aranhas e escorpiões, também ocorre doença de chagas, ferradas por arraias nas épocas que surgem as praias e outros tipos de intercorrências peculiares a esta região (REIS MHS, et al., 2019).

Diversos desafios surgiram na área ribeirinha com o impacto da pandemia de Covid-19, neste sentido, o planejamento e montagem de estratégias foi primordial para assistir esses povos, começando pela testagem em massa dos casos de coronavírus até a imunização desses indivíduos (JÚNIOR JCFP, et al., 2020). Ações que tiveram parcerias intersetoriais essenciais, justamente em uma época que abalou bastante o emocional de todas as classes profissionais, visto que desde a descoberta dos primeiros casos até os picos principais da pandemia, diversos profissionais morreram ou perderam alguém próximo (PORTUGAL JKA, et al., 2020).

Outro agravo que tem ganhado destaque entre essa população é a automedicação, prática de consumir medicamentos farmacêuticos sem prescrição, principalmente antibióticos, informação que inclusive é confirmada por estudo da Universidade de São Paulo (USP), envolvendo ribeirinhos locais (GAMA ASM, 2016). Pelo fato de residirem isolados da sede municipal, quem mora na zona rural utiliza de recursos e culturas locais no tratamento de enfermidades, assim como os povos indígenas que possuem suas crenças e heranças culturais herdadas de seus antepassados, principalmente no uso de plantas e ervas medicinais no combate a determinadas doenças (GAMA ASM, et al., 2018).

Considerando as peculiaridades das extensas áreas rurais do município, sugere-se o estudo da viabilidade econômica da UBS fluvial e a possibilidade de implantação de polos bases de saúde, similares ao modelo implantado em Distritos Sanitários Indígenas (DSEI), possibilitando atendimento contínuo e talvez a redução dos custos na operacionalização das ações de saúde. Além disto, as populações ribeirinhas locais vivem em áreas isoladas e de difícil acesso, as quais limitam a busca e acesso aos serviços de saúde, nesta perspectiva, é possível visualizar avanços na assistência à saúde destes povos através da implantação de estratégias de cobertura da saúde com a UBS fluvial e ações conjuntas entre diferentes instituições (Universidades e Governo Municipal).

\section{REFERÊNCIAS}

1. BRASIL. Lei no 8.080, de 19 de setembro de 1990. Lei Orgânica da Saúde. Dispõe sobre as condições para a promoção, proteção e recuperação da saúde, a organização e o funcionamento dos serviços correspondentes e dá outras providências. Brasília, set. 1990. Disponível em: http://www.planalto.gov.br/ccivil_03/leis/l8080.html. Acesso em: 03 nov. 2021.

2. CAMPOS AG, CHAVES JV. Seguro defeso: diagnóstico dos problemas enfrentados pelo programa. Brasília: Ipea, 2014.

3. DIEGUES AC, et al. Os Saberes Tradicionais e a Biodiversidade no Brasil. 1ª ed. São Paulo: Probio-MMA, 2000; $211 \mathrm{p}$.

4. FRANCO EC, et al. Promoção da saúde da população ribeirinha da região amazônica: relato de experiência. Rev CEFAC, 2015.

5. FRAXE TJP, et al. Comunidades ribeirinhas amazônicas: modos de vida e uso dos recursos naturais. Manaus: EDUA; 2007.

6. GAMA ASM. Automedicação em comunidades ribeirinhas na região do Médio Solimões, Amazonas. Tese (Doutorado em Enfermagem na Saúde do Adulto). Universidade de São Paulo (USP), São Paulo, 2016; 115 p.

7. GAMA ASM, et al. Inquérito de saúde em comunidades ribeirinhas do Amazonas, Brasil. Cad. Saúde Pública, 2018. 
8. GUIMARÃES AF, et al., Acesso a serviços de saúde por ribeirinhos de um município no interior do estado do Amazonas, Brasil. Rev Pan Amaz Saude, 2020.

9. INSTITUTO BRASILEIRO DE GEOGRAFIA E ESTATÍSTICA (IBGE). Sinopse do censo demográfico de 2010.

10. JÚNIOR JCFP, et al. Atuação de acadêmicos de enfermagem nas ações de enfrentamento da covid-19 em comunidades ribeirinhas do amazonas: relato de experiência. Revista eletrônica Acervo Saúde, 2020.

11. MINISTÉRIO DA SAÚDE. Política Nacional de Atenção Básica (PNAB). Série E. Legislação em Saúde. Brasília - DF: Brasil, 2012 Brasília: Ministério da Saúde, 2012. Disponível em: http://189.28.128.100/dab/docs/publicacoes/geral/pnab.pdf. Acesso em: 03 nov. 2021.

12. MINISTÉRIO DA SAÚDE. Portaria ํㅜ 2.488, de 21 de outubro de 2011. Aprova a Política Nacional de Atenção Básica, estabelecendo a revisão de diretrizes e normas para a organização da Atenção Básica, para a Estratégia Saúde da Família (ESF) e o Programa de Agentes Comunitários de Saúde (PACS). Diário Oficial [da] República Federativa do Brasil. Brasília - DF: 2011a. Brasil, Disponível em: https://bvsms.saude.gov.br/bvs/saudelegis/gm/2011/prt2488_21_10_2011.html. Acesso em: 03 nov. 2021.

13. MINISTÉRIO DA SAÚDE. Portaria nำ2.490, de 21 de outubro de 2011. Define os valores de financiamento das Equipes de Saúde da Família Ribeirinhas (ESFR) e custeio das Unidades Básicas de Saúde Fluviais (UBSF), mediante a revisão de diretrizes e normas para a organização da Atenção Básica, instituídos pela Política Nacional de Atenção Básica. Diário Oficial [da] República Federativa do Brasil. Brasília - DF: Brasil, 2011b. Disponível em: https://bvsms.saude.gov.br/bvs/saudelegis/gm/2011/prt2490_21_10_2011.html. Acesso em: 03 nov. 2021.

14. MINISTÉRIO DA SAÚDE. Portaria no 2.436, de 21 de setembro de 2017. Aprova a Política Nacional de Atenção Básica, estabelecendo a revisão de diretrizes para a organização da Atenção Básica, no âmbito do Sistema Único de Saúde (SUS). Brasília - $\quad$ DF: Brasil, 2017. Disponível em: https://bvsms.saude.gov.br/bvs/saudelegis/gm/2017/prt2436_22_09_2017.html. Acesso em: 03 nov. 2021.

15. MINISTÉRIO DA SAÚDE. Programa agentes comunitários de saúde (PACS). Brasília - DF: Brasil, 2001. Ministério da Saúde. Disponível em: https://bvsms.saude.gov.br/bvs/publicacoes/pacs01.pdf. Acesso em: 03 nov. 2021.

16. PORTUGAL JKA, et al. Percepção do impacto emocional da equipe de enfermagem diante da pandemia de COVID19: relato de experiência. Revista eletrônica Acervo Saúde, 2020; Esp. 46: e3794.

17. REIS MHS, et al. Ações de saúde em populações ribeirinhas no interior do estado do Amazonas: Relato de experiência. In: Schweickardt JC, Atenção Básica na região amazônica: Saberes e práticas para o fortalecimento do SUS. Porto Alegre: Rede Unida, 2009; 171-181.

18. REIS MHS, et al. O impacto do advento de uma Unidade Básica de Saúde Fluvial na assistência aos povos ribeirinhos do Amazonas. Revista eletrônica Acervo Saúde, 2020; Sup.53; e3631.

19. SANTOS FS, et al. Prevalência de enteroparasitismo em crianças de comunidades ribeirinhas do Município de Coari, no médio Solimões, Amazonas, Brasil. Rev Pan Amaz Saude. 2010.

20. SOUSA IS. As condições de vida e saneamento nas comunidades da área de influência do gasoduto Coari-Manaus em Manacapuru - AM. Hygeia. 2009; 5(9): 88-98. 